Pacific

Journal of

Mathematics

CLASSIFICATION OF SOLUTIONS OF CERTAIN FOURTH-ORDER NONLINEAR ELLIPTIC EQUATIONS IN $\mathbb{R}^{4}$

XINGWANG XU

Volume $225 \quad$ No. 2

June 2006 


\title{
CLASSIFICATION OF SOLUTIONS OF CERTAIN FOURTH-ORDER NONLINEAR ELLIPTIC EQUATIONS IN $\mathbb{R}^{4}$
}

\author{
XINGWANG XU
}

\begin{abstract}
We consider the uniqueness of solutions of the equation $\Delta^{2} u=e^{u}$ in fourdimensional Euclidean space. Our main result is that the solutions are all classical ones, provided that the energy of the solutions is finite and the diffusion of the solutions decays to zero at infinity. The method we used in this paper is known as the method of moving spheres.
\end{abstract}

\section{Introduction}

We consider the fourth-order nonlinear elliptic differential equation

$$
\Delta^{2} u=e^{u} \quad \text { in } \mathbb{R}^{4} .
$$

It is easy to check that for all $\varepsilon>0$ and $x_{0} \in \mathbb{R}^{4}$, the functions

$$
u(x)=\log \frac{384 \varepsilon^{2}}{\left(\varepsilon+\left|x-x_{0}\right|^{2}\right)^{4}},
$$

where $\varepsilon$ and $x_{0}$ are parameters, are solutions of (1-1). An easy computation shows that these functions satisfy

$$
\int_{\mathbb{R}^{4}} e^{u} d x=B<\infty
$$

and

$$
\lim _{|x| \rightarrow \infty} \Delta u=0,
$$

where $B=32 \omega_{4}$, the volume of the unit sphere in $\mathbb{R}^{4}$ being denoted by $\omega_{4}$.

We are interested in the classification of solutions of (1-1) under conditions (1-3) and (1-4), for arbitrary $B$. We prove:

Main Theorem. Let $u \in C^{4}\left(\mathbb{R}^{4}\right)$ be a solution of (1-1) satisfying the additional conditions (1-3) and (1-4) for some constant $B>0$. Then $u$ takes the form (1-2) for some $\varepsilon>0$ and $x_{0} \in \mathbb{R}^{4}$.

MSC2000: primary 35J60, 58G35; secondary 53C21.

Keywords: conformally invariant equation, symmetry, moving sphere method. 
Equation (1-1) has roots in conformal geometry. Let $(M, g)$ be a complete Riemmanian manifold. Associated to $g$, there are tensors such as the full curvature tensor $R$, the Ricci curvature tensor Ric and the scalar curvature $s$. The Laplace operator $\Delta$ is a well known elliptic operator on $M$ associated with the metric $g$. In dimension 4, the integrand of Gauss-Bonnet formula modulo the Weyl tensor part is given by

$$
12 Q=-\Delta s+s^{2}-3 \operatorname{Ric}^{2},
$$

where the $Q$-curvature $Q$ is similar to the scalar curvature in dimension 2 . From the conformal geometric point of view, consider two metrics $g, g_{0}$ related by $g=e^{2 w} g_{0}$. If $Q_{g}$ and $Q_{g_{0}}$ are the respective $Q$-curvatures, one checks by an easy calculation that

$$
P w+2 Q_{0}=Q_{g} e^{4 w},
$$

where $P=\Delta_{g_{0}}^{2}+\delta\left(\frac{2}{3} s I-2\right.$ Ric $) d$ is the Paneitz operator. Once we consider $M=\mathbb{R}^{4}$ and $g_{0}=\delta_{i j}$, the standard metric on $\mathbb{R}^{4}$, the preceding equation takes the form:

$$
\Delta^{2} w=Q_{g} e^{4 w} .
$$

We are interested in the problem of classifying the solutions of (1-5) with $Q_{g}$ constant. By simple rescaling, we can reduce (1-5) to (1-1).

It is also known that the operator $P$ is conformally covariant and $S^{4}$ and $\mathbb{R}^{4}$ are conformally related. On $S^{4}$, Equation (1-5) takes the form

$$
\Delta^{2} v-2 \Delta v=6\left(e^{4 v}-1\right),
$$

if we assume that $Q_{g}=Q_{g_{0}}=3$. From the conformal point of view, we are interested in (1-6) rather than (1-5). Under stereographic projection, if $v$ is a solution of (1-6), then $u=v+\log 2-\log \left(1+r^{2}\right)$ will be a solution of (1-5) satisfying (1-3) and (1-4). Hence we have shown:

Corollary. All solutions of (1-6) arise from conformal transformations of $\mathrm{S}^{4}$ (such solutions are known as classical).

The statement that all solutions of (1-6) with minimizing energy are classical solutions was proved by Chang and Yang [1995] and the general case was announced by M. Gursky, with a proof along very different lines.

In this work we use the method of moving spheres, a variant of the method of moving planes. The latter is well known and has been put to use in the classification of certain nonlinear second order elliptic equations; see [Caffarelli et al. 1989; Gidas et al. 1979; Chen and Li 1991], for example. The method of moving spheres is relatively new; see [Li and Zhu 1995] and its references. The main ingredient in the method is the maximum principle. However, the equation under consideration 
is of order four, so the principle does not hold. This is the fundamental difficulty in dealing with this equation.

The paper is organized as follows. In Section 2 we prove that there is an upper bound for all solutions of (1-1) under assumptions (1-3) and (1-4). In fact, condition (1-4) is not necessary for this purpose. After we have this important property of the solutions, we derive in Section 3 other properties of the solutions to be used later. The crux of the work is Section 4, where we prove the main theorem.

A similar problem can be posed for higher-dimensional spaces. We will come back to it in future publications.

Remark. Similar results have been obtained in [Chang and Yang 1997] and [Lin 1998] using the method of moving planes.

\section{An upper bound for $u$}

Theorem 2.1. If $u \in C^{4}\left(\mathbb{R}^{4}\right)$ is a solution of (1-1) satisfying (1-3) and (1-4) for some constant $B>0$, there exists a constant $C>0$ such that $\sup _{\mathbb{R}^{4}} u<C$.

Lemma 2.2. If $u$ satisfies (1-1) and (1-3), then

$$
\Delta u \leq 0 .
$$

Proof. We want to show that $\Delta u\left(x_{0}\right) \leq 0$ for any $x_{0} \in \mathbb{R}^{4}$. By a change of coordinates, we can assume $x_{0}=0$. Set

$$
\bar{u}(r)=\frac{1}{\omega_{4} r^{3}} \int_{|x|=r} u d s \quad \text { and } \quad \bar{w}(r)=\frac{1}{\omega_{4} r^{3}} \int_{|x|=r} w d s,
$$

where $w=-\Delta u$. Since $\bar{u}(0)=u(0)$ and $\bar{w}(0)=w(0)$, we only need to show that $w(0) \geq 0$. By the divergence theorem, we know that

$$
\int_{B_{\rho}} \Delta w d x=\rho^{3} \frac{\partial}{\partial \rho}\left(\rho^{-3} \int_{\partial B_{\rho}} w d s\right)
$$

(see [Gilbarg and Trudinger 1983, p. 14]). But in our case, $\Delta w=-e^{u}$. Hence,

$$
\frac{\partial}{\partial \rho}\left(\rho^{-3} \int_{\partial B_{\rho}} w d s\right) \leq 0
$$

Integrate both sides from 0 to $R$ to get

$$
R^{-3} \int_{\partial B_{R}} w d s \leq \omega_{4} w(0)
$$

for all $R>0$. 
Now, on the contrary, assume that $w(0)<0$. Thus for all $r>0$,

$$
\bar{w}(r)=\frac{1}{\omega_{4} r^{3}} \int_{|x|=r} w d s \leq w(0)<0 .
$$

On the other hand,

$$
\bar{u}^{\prime \prime}(r)+\frac{3}{r} \bar{u}^{\prime}(r)+\bar{w}(r)=0,
$$

so we obtain the inequality

$$
\bar{u}^{\prime \prime}(r)+\frac{3}{r} \bar{u}^{\prime}(r) \geq-w(0) .
$$

Multiplying by $r^{3}$, integrating, and using the fact that $\bar{u}^{\prime}(r)$ is bounded near $r=0$, we obtain

$$
r^{3} \bar{u}^{\prime}(r) \geq-\frac{1}{4} w(0) r^{4} .
$$

Thus we get $\bar{u}^{\prime}(r) \geq-\frac{1}{4} w(0) r$. It follows that

$$
\bar{u}(r) \geq u(0)-\frac{1}{8} w(0) r^{2} .
$$

Since $\omega_{4} \int_{0}^{R} r^{3} \bar{u}(r) d r=\int_{B_{R}} u d x$, thus

$$
\omega_{4} \int_{0}^{\infty} r^{3} \bar{u}(r) d r=\int_{\mathbb{R}^{4}} u d x \leq \int_{\mathbb{R}^{4}} e^{u} d x<B<\infty .
$$

Now since $-w(0)>0$, there is a contradiction between the last two displayed equations. Therefore we have shown that $w(0) \geq 0$, as desired.

Proof of Theorem 2.1. By assumption (1-4), there exists a constant $A>0$ such that

$$
\Delta u \geq-A \quad \text { in } \mathbb{R}^{4} .
$$

From Lemma 2.2, then, we have $|f| \leq A$ on $\mathbb{R}^{4}$, where $f$ is defined by $f(x)=$ $-\Delta u(x)$. Given $x_{0} \in \mathbb{R}^{4}$, let $B_{1}$ be a ball of radius 1 centered at $x_{0}$. Recalling that $\Delta u \in C^{2}\left(\mathbb{R}^{4}\right)$, we take $u_{1}$ to be a solution of

$$
\begin{cases}-\Delta u_{1}=f & \text { in } B_{1}, \\ u_{1}=0 & \text { on } \partial B_{1} .\end{cases}
$$

From elliptic theory we know that $\left|u_{1}\right| \leq C_{1}$, for some constant $C_{1}$ depending on $A$ but not depending on $x_{0}$. Set $u_{2}=u-u_{1}$. Then $\Delta u_{2}=0$ in the ball $B_{1}$. By the mean value theorem for harmonic functions, we have

$$
\left\|u_{2}^{+}\right\|_{L^{\infty}\left(B_{1 / 2}\right)} \leq C_{2} \int_{B_{1}} u_{2}^{+} d x .
$$


On the other hand, we have $u_{2}^{+} \leq u^{+}+\left|u_{1}\right|$, and since

$$
\int_{\mathbb{R}^{4}} u^{+} d x \leq \int_{\mathbb{R}^{4}} e^{u} d x=B<+\infty
$$

by assumption (1-3), we see that $\left\|u_{2}^{+}\right\|_{L^{1}\left(B_{1}\right)} \leq C_{3}(A, B)$. Combining this with (2-1), we obtain $\left\|u_{2}^{+}\right\|_{L^{\infty}\left(B_{1 / 2}\right)} \leq C_{4}$. Finally, since $u=u_{2}+u_{1}$, we get

$$
u^{+} \leq u_{2}^{+}+\left|u_{1}\right| \leq C_{5}(A, B),
$$

as desired.

Remark. In fact, Theorem 2.1 holds even without assumption (1-4), but we do not need this fact.

\section{Decay properties of the solutions at infinity}

Theorem 3.1. Suppose that $u \in C^{4}\left(\mathbb{R}^{4}\right)$ is a solution of (1-1) satisfying (1-3) and (1-4) for some constant $B>0$. Set

$$
d=\frac{1}{4 \omega_{4}} \int_{\mathbb{R}^{4}} e^{u} d x
$$

Then

$$
\begin{aligned}
\Delta u(x) & =-\frac{1}{2 \omega_{4}} \int_{\mathbb{R}^{4}} \frac{e^{u}}{|x-y|^{2}} d y, \\
4 x \cdot \nabla u(x) & \geq|x|^{2} \Delta u(x)-2 d, \\
\left|\frac{u(x)}{\log |x|}\right| & \leq C \quad \text { for some constant } C \text { and }|x| \text { large enough. }
\end{aligned}
$$

Proof. Consider the function $w$ defined by

$$
w(x)=\frac{1}{4 \omega_{4}} \int_{\mathbb{R}^{4}}(\log |x-y|-\log |y|) e^{u(y)} d y .
$$

A simple calculation shows that

$$
\Delta w(x)=\frac{1}{2 \omega_{4}} \int_{\mathbb{R}^{4}} \frac{e^{u}}{|x-y|^{2}} d y \quad \text { and } \quad \Delta^{2} w=-e^{u} .
$$


Now, since $u \leq C$ for some constant $C$ by Theorem 2.1, we have, for all $x \in \mathbb{R}^{4}$,

$$
\begin{aligned}
|\Delta w(x)| & =\frac{1}{2 \omega_{4}} \int_{\mathbb{R}^{4}} \frac{e^{u}}{|x-y|^{2}} d y \\
& \leq \frac{1}{2 \omega_{4}} \int_{|x-y| \leq 1} \frac{e^{u}}{|x-y|^{2}} d y+\frac{1}{2 \omega_{4}} \int_{|x-y| \geq 1} \frac{e^{u}}{|x-y|^{2}} d y \\
& \leq \frac{e^{C}}{2 \omega_{4}}\left(\omega_{4} \int_{0}^{1} r d r\right)+\frac{1}{2 \omega_{4}} \int_{|x-y| \geq 1} e^{u} d y \\
& \leq \frac{1}{4} e^{C}+2 d \leq C_{6} .
\end{aligned}
$$

Let $\varphi=u+w$. Clearly,

$$
\Delta^{2} \varphi=\Delta^{2} u+\Delta^{2} w=e^{u}-e^{u}=0
$$

and

$$
-A \leq \Delta \varphi=\Delta u+\Delta w \leq C_{6},
$$

since $\Delta u \leq 0$ by Lemma 2.2 .

Interior gradient estimates show that $\Delta \varphi$ is a constant. But we can see, from assumption (1-4) and Theorem 2.1, that $\lim _{|x| \rightarrow \infty} \Delta \varphi=0$. Thus (i) is proved.

Now from (i), we know that $\varphi$ is a harmonic function. As a first step, for large $x$, we have

$$
\begin{aligned}
\left|\frac{w(x)}{\log |x|}-d\right| & =\frac{1}{4 \omega_{4} \log |x|}\left|\int_{\mathbb{R}^{4}}(\log |x-y|-\log |x|-\log |y|) e^{u} d y\right| \\
& \leq \frac{1}{4 \omega_{4} \log |x|} \int_{|y| \leq 1}\left|\log \frac{|x-y|}{|x||y|}\right| e^{u} d y \\
& +\frac{1}{4 \omega_{4} \log |x|} \int_{|y| \geq 1}\left|\log \frac{|x-y|}{|x||y|}\right| e^{u} d y \leq C_{1} .
\end{aligned}
$$

Here we have used the fact that the function $\log |x-y|$ is integrable over the ball $B(x, 1)$.

Since $u$ has an upper bound by Theorem 2.1, we conclude there are constants $\alpha, \beta$ such that $\varphi(x) \leq \alpha+\beta \log |x|$. Hence $\varphi(x) \leq \alpha+\beta \log \left(\left|x-x_{0}\right|+\left|x_{0}\right|\right)$, for any $x_{0} \in \mathbb{R}^{4}$ and any $r=\left|x-x_{0}\right|>0$.

On $B_{r}\left(x_{0}\right)$, the ball of radius $r$ at $x_{0}$, we consider $\psi(x)=\alpha+\beta \log \left(r+\left|x_{0}\right|\right)-\varphi$. It is easy to see that $\psi(x) \geq 0$ in the ball $B_{r}\left(x_{0}\right)$ and $\Delta \psi=0$. For $i=1,2,3$, 4, we have $\Delta\left(\partial \psi / \partial x_{i}\right)=0$ in the ball $B_{r}\left(x_{0}\right)$. By the mean value theorem for harmonic 
functions we then have, for $0 \leq \rho \leq r$,

$$
\begin{aligned}
\left|\frac{\partial \varphi}{\partial x_{i}}\left(x_{0}\right)\right| & =\left|\frac{\partial \psi}{\partial x_{i}}\left(x_{0}\right)\right|=\left|\frac{1}{\omega_{4} \rho^{4}} \int_{\left|x-x_{0}\right| \leq \rho} \frac{\partial \psi}{\partial x_{i}}(x) d x\right| \\
& =\frac{1}{\omega_{4} \rho^{4}}\left|\int_{\left|x-x_{0}\right|=\rho} \psi(x) \frac{x_{i}-\left(x_{0}\right)_{i}}{\rho} d s\right| \\
& \leq \frac{1}{\omega_{4} \rho^{4}} \int_{\left|x-x_{0}\right|=\rho} \psi(x) d s=\frac{1}{\rho}\left(\alpha+\beta \log \left(r+\left|x_{0}\right|\right)-\varphi\left(x_{0}\right)\right) .
\end{aligned}
$$

If we fix $x_{0}$, set $\rho=r$ and let $r$ tend to infinite, we see that $\varphi$ is a constant. Thus $u=C-w(x)$.

Hence relation (iii) follows from (3-4). To show (ii), we work as follows:

$$
\begin{aligned}
4|x \cdot \nabla u| & =4\left|-\frac{1}{4 \omega_{4}} \int_{\mathbb{R}^{4}} \frac{x \cdot(x-y)}{|x-y|^{2}} e^{u} d y\right| \leq \frac{|x|}{\omega_{4}} \int_{\mathbb{R}^{4}} \frac{e^{u}}{|x-y|} d y \\
& \leq \frac{|x|}{\omega_{4}}\left(\int_{\mathbb{R}^{4}} \frac{e^{u}}{|x-y|^{2}} d y\right)^{1 / 2}\left(\int_{\mathbb{R}^{4}} e^{u} d y\right)^{1 / 2} \\
& =2 \sqrt{2}\left(|x|^{2}(-\Delta u)\right)^{1 / 2} d^{1 / 2} \leq|x|^{2}(-\Delta u)+2 d .
\end{aligned}
$$

Remark. For getting (i), we can relax condition (1-4) to $\lim _{|x| \rightarrow \infty} \sup \Delta u=0$. Once we obtain (i), inequality (ii) follows with the same argument.

\section{Proof of Theorem 2.1}

We start with an observation easily checked by an elementary calculation. Let $u \in C^{4}\left(\mathbb{R}^{4}\right)$ satisfy $(1-1)$. If we set $v(x)=u\left(x /|x|^{2}\right)-8 \log |x|$, the function $v$ satisfies

$$
\Delta^{2} v=\exp v
$$

Similarly, for $\lambda>0$, set

$$
v_{\lambda}(x)=v\left(\frac{\lambda^{2} x}{|x|^{2}}\right)-8 \log \frac{|x|}{\lambda} .
$$

Then $v_{\lambda}(x)=u\left(x / \lambda^{2}\right)-8 \log \lambda$ and hence $\Delta^{2} v_{\lambda}=\exp v_{\lambda}$. Combining this with $(4-1)$, we get:

Lemma 4.1. Let $v$ and $v_{\lambda}$ be as above. The function $w_{\lambda}:=v-v_{\lambda}$ satisfies

$$
\Delta^{2} w_{\lambda}(x)=(\exp \psi(x)) w_{\lambda}(x),
$$

where $\psi(x)$ is a real number between $v(x)$ and $v_{\lambda}(x)$. 
Proposition 4.2. For $\lambda>0$ large enough, $w_{\lambda}(x)$ and $-\Delta w_{\lambda}(x)$ are nonnegative for all $x \in B_{\lambda}(0) \backslash\{0\}$.

Proof. Step 1. There exists $R_{0}>0$ such that $w_{\lambda}(x) \geq 0$ for $R_{0} \leq|x| \leq \lambda / 2$. Indeed,

$$
\begin{aligned}
w_{\lambda}(x) & =v(x)-v\left(\frac{\lambda^{2} x}{|x|^{2}}\right)+8 \log \frac{|x|}{\lambda}=u\left(\frac{x}{|x|^{2}}\right)-u\left(\frac{x}{\lambda^{2}}\right)-8 \log \frac{|x|}{\lambda} \\
& =O\left(\frac{1}{|x|}\right)+8 \log \frac{\lambda}{|x|} \geq O\left(\frac{1}{|x|}\right)+8 \log 2,
\end{aligned}
$$

which is positive if $R_{0}$ is large enough.

Step 2. There exists $R_{1}>R_{0}$ such that $w_{\lambda}(x) \geq 0$ and $\Delta w_{\lambda}(x) \leq 0$ for $R_{1} \leq|x| \leq \lambda$. Indeed,

$$
\begin{aligned}
-\Delta w_{\lambda} & =\Delta\left(u\left(\frac{x}{\lambda^{2}}\right)\right)-\Delta\left(u\left(\frac{x}{|x|^{2}}\right)\right)+8 \Delta(\log |x|) \\
& =\frac{1}{|x|^{2}}\left(\frac{|x|^{2}}{\lambda^{4}}(\Delta u)\left(\frac{x}{\lambda^{2}}\right)-\frac{1}{|x|^{2}}(\Delta u)\left(\frac{x}{|x|^{2}}\right)+4 \sum_{i=1}^{4} \frac{x_{i}}{|x|^{2}} u_{i}\left(\frac{x}{|x|^{2}}\right)+16\right) .
\end{aligned}
$$

If $R_{1}>1$, we have

$$
\left|\frac{x}{|x|^{2}}\right|=\frac{1}{|x|} \leq \frac{1}{R_{1}} \leq 1 \quad \text { if }|x| \geq R_{1} .
$$

Set $C=\max _{\bar{B}_{1}}|\nabla u|$. Then

$$
\left|4 \sum_{i=1}^{4}\left(\frac{x_{i}}{|x|^{2}}\right) u_{i}\left(\frac{x}{|x|^{2}}\right)\right| \leq \frac{4 C}{|x|} \leq 1
$$

if $R_{1}$ is large enough. Letting $B=\max _{\bar{B}_{1}}|\Delta u|$, we get

$$
\left.\left|\frac{|x|^{2}}{\lambda^{4}}(\Delta u)\left(\frac{x}{\lambda^{2}}\right)-\right| x\right|^{-2}(\Delta u)\left(\frac{x}{|x|^{2}}\right) \mid \leq \frac{|x|^{2}}{\lambda^{4}} B+\frac{1}{|x|^{2}} B \leq\left(\frac{1}{\lambda^{2}}+\frac{1}{|x|^{2}}\right) B \leq 4
$$

if $B / R_{1}<1$. Therefore, if $R_{1} \geq R_{0}$ is large enough, we have $-\Delta w_{\lambda}(x) \geq 0$ for $R_{1} \leq|x| \leq \lambda$.

Since $w_{\lambda}(x) \geq 0$ when $|x|=\lambda / 2$ and $w_{\lambda}(x)=0$ when $|x|=\lambda$, we conclude by the maximum principle that $w_{\lambda} \geq 0$ in the region $R_{1} \leq \lambda / 2 \leq|x| \leq \lambda$.

Step 3. If $d=\left(1 / 4 \omega_{4}\right) \int_{\mathbb{R}^{4}} e^{u} d x<8$, there exists $R_{2} \geq R_{1}$ such that $w_{\lambda}(x) \geq 0$ and $\overline{-\Delta w_{\lambda}} \geq 0$ for all $x \in B_{R_{0}}(0) \backslash\{0\}$ and all $\lambda \geq R_{2}$. Indeed, for $0<|x| \leq R_{0}, d<8$, 
and $\lambda$ large enough, we have

$$
\begin{aligned}
w_{\lambda}(x) & =v(x)-\left(v\left(\frac{\lambda^{2} x}{|x|^{2}}\right)-8 \log \frac{|x|}{\lambda}\right)=u\left(\frac{x}{|x|^{2}}\right)-u\left(\frac{x}{\lambda^{2}}\right)-8 \log \frac{|x|}{\lambda} \\
& =\left(u\left(\frac{x}{|x|^{2}}\right)-8 \log |x|\right)-u\left(\frac{x}{\lambda^{2}}\right)+8 \log \lambda .
\end{aligned}
$$

Since $u\left(x /|x|^{2}\right)-8 \log |x|=(d-8+o(1)) \log |x|$ remains positive as $x$ tends to 0 , we see that

$$
u\left(\frac{x}{|x|^{2}}\right)-8 \log |x| \geq-C \quad \text { for } x \in B_{R_{0}}(0) \backslash\{0\}
$$

and that $u\left(x / \lambda^{2}\right) \leq C_{1}$; thus, if $\lambda$ is large enough, we have $w_{\lambda}(x) \geq 0$ for $x \in$ $B_{R_{0}}(0) \backslash\{0\}$. Similarly,

$$
\begin{aligned}
|x|^{2}(- & \left.\Delta w_{\lambda}(x)\right) \\
& =\frac{|x|^{2}}{\lambda^{4}}(\Delta u)\left(\frac{x}{\lambda^{2}}\right)-\frac{1}{|x|^{2}}(\Delta u)\left(\frac{x}{|x|^{2}}\right)+4\left(\frac{x}{|x|^{2}} \cdot \nabla u\left(\frac{x}{|x|^{2}}\right)\right)+16 \\
& \geq 2(8-d)+\frac{|x|^{2}}{\lambda^{4}}(\Delta u)\left(\frac{x}{\lambda^{2}}\right),
\end{aligned}
$$

where we have used Theorem 3.1(ii). Now observe that when $\lambda$ is large,

$$
\frac{|x|^{2}}{\lambda^{4}}(\Delta u)\left(\frac{x}{\lambda^{2}}\right)
$$

can be as small as we want. Thus we conclude that $\left(-\Delta w_{\lambda}(x)\right) \geq 0$ for $x \in$ $B_{R_{0}}(0) \backslash\{0\}$.

Together the three steps imply that the proposition is true, apart form the claim that $-\Delta w_{\lambda}(x) \geq 0$ in the region $R_{0} \leq|x| \leq \lambda / 2$. But it is easy to verify this by using the maximum principle, since $\Delta\left(-\Delta w_{\lambda}(x)\right)=-(\exp \psi(x)) w_{\lambda}(x) \leq 0$ and $-\Delta w_{\lambda}(x) \geq 0$ on $|x|=R_{0} / 2$ by Step 3 and $-\Delta w_{\lambda}(x) \geq 0$ on $|x|=3 \lambda / 4$ by Step 2.

Now we define, for $b \in \mathbb{R}^{4}$,

$\lambda_{b}=\inf \left\{\lambda>0: w_{\mu, b}(x) \geq 0,-\Delta w_{\mu, b}(x) \geq 0\right.$ in $B_{\mu}(0) \backslash\{0\}$ for $\left.\lambda<\mu<+\infty\right\}$, where $w_{\lambda, b}=v_{b}(x)-\left(v_{b}\right)_{\lambda}(x), v_{b}(x)=u_{b}\left(x /|x|^{2}\right)-8 \log |x|$ and $u_{b}(x)=u(x+b)$.

Proposition 4.3. There exists $\bar{b} \in \mathbb{R}^{4}$ such that $\lambda_{\bar{b}}>0$. 
Proof. For all $b \in \mathbb{R}^{4}, \lambda>0$, set

$$
g_{b, \lambda}(x)=v_{b}(x)-\left(v_{b}\left(\frac{\lambda^{2} x}{|x|^{2}}\right)-8 \log \frac{|x|}{\lambda}\right)=w_{\lambda, b}(x)
$$

for all $x \in B_{\lambda}(0) \backslash\{0\}$.

Suppose the proposition is not true. Then $w_{\lambda, b} \geq 0$ for all $b \in \mathbb{R}^{4}$ and all $\lambda>0$.

Now, one observes that

$$
\begin{aligned}
g_{b,|x|}(x) & =0, \\
g_{b,|x|}(r x) & =8 \log r+v_{b}(r x)-v_{b}\left(\frac{x}{r}\right) \geq 0 \quad \text { for all } 0<r<1 .
\end{aligned}
$$

It follows that

$$
\left.\frac{d}{d r}\left(g_{b,|x|}(r x)\right)\right|_{r=1} \leq 0, \quad \text { that is, } \quad \frac{8}{r}+\left(\nabla v_{b}\right)(r x) \cdot x+\left.(\nabla v)\left(\frac{x}{r}\right) \cdot \frac{x}{r^{2}}\right|_{r=1} \leq 0 .
$$

Simplify this to get

$$
8+2 \nabla v_{b} \cdot x \leq 0
$$

Now by a change of variables we get

$$
(x-b) \cdot(\nabla v)(x)+4 \leq 0 .
$$

Dividing both sides by $|b|$ and sending $|b|$ to $+\infty$, we have

$$
-\nabla v \cdot \omega_{b} \leq 0 .
$$

Thus $-\nabla v(x) \cdot \omega_{b} \geq 0$ for all $y \in \mathbb{R}^{4}$. Thus from (19) and arbitrarily of $b$, we have $\nabla v=0$. Thus $v$ is a constant, contradicting the fact that $\int_{\mathbb{R}^{4}} e^{v} d x=\int_{\mathbb{R}^{4}} e^{u} d x<\infty$.

Proposition 4.4. Suppose there exists $b \in \mathbb{R}^{4}$ such that $\lambda_{b}>0$. Then $w_{\lambda, b}(x)$ vanishes for all $x \in \mathbb{R}^{4}$.

Proof. Assume without loss of generality that $b=0$. Suppose, contrary to the proposition, that some $w_{\lambda_{0}}(x) \not \equiv 0$ satisfies

$$
\begin{array}{cc}
w_{\lambda_{0}} \geq 0 \text { and }-\Delta w_{\lambda_{0}} \geq 0 & \text { in } B_{\lambda_{0}} \backslash\{0\}, \\
\Delta^{2} w_{\lambda_{0}}=e^{\psi} w_{\lambda_{0}} & \text { in } B_{\lambda_{0}} \backslash\{0\} .
\end{array}
$$

Recall that

$$
\begin{aligned}
w_{\lambda_{0}}(x) & =u\left(\frac{x}{|x|^{2}}\right)-u\left(\frac{x}{\lambda_{0}^{2}}\right)+8 \log \lambda_{0}-8 \log |x| \\
& =u\left(\frac{x}{|x|^{2}}\right)-8 \log |x|+8 \log \lambda_{0}-u\left(\frac{x}{\lambda_{0}^{2}}\right) .
\end{aligned}
$$


Set $\delta=8 \log \lambda_{0}-C$, where $C$ is the upper bound of $u$.

$$
u\left(\frac{x}{|x|^{2}}\right)-8 \log |x|=(d-8+o(1)) \log |x|
$$

remains positive as $|x| \rightarrow 0$, we can choose $0<r_{0}<1$ such that

$$
u\left(\frac{x}{|x|^{2}}\right)-8 \log |x| \geq \frac{d-8}{2} \log |x| \geq \frac{d-8}{2} \log r_{0} \geq 1-\delta
$$

if $|x| \leq r_{0}$. Therefore, if $|x| \leq r_{0} / 2$,

$$
\begin{aligned}
w_{\lambda_{0}}(x) & =u\left(\frac{x}{|x|^{2}}\right)-u\left(\frac{x}{\lambda_{0}^{2}}\right)+8 \log \lambda_{0}-8 \log |x| \\
& \geq u\left(\frac{x}{|x|^{2}}\right)+8 \log \lambda_{0}-8 \log |x|-C \geq 1>0 .
\end{aligned}
$$

and similarly, by using equation (4-2), we can easily see that there exists a $r_{0}>0$ such that $-\Delta w_{\lambda_{0}}(x) \geq 1$ if $|x| \leq r_{0} / 2$.

Now consider that

$$
\begin{array}{cl}
\Delta^{2} w_{\lambda_{0}}=e^{\psi} w_{\lambda_{0}} & \text { in } B_{\lambda_{0}} \backslash B_{r_{0} / 2}(0), \\
w_{\lambda_{0}}>0 \text { and }-\Delta w_{\lambda_{0}} \geq 0 & \text { in } B_{\lambda_{0}} \backslash B_{r_{0} / 2}(0), \\
w_{\lambda_{0}}>0 \text { and }-\Delta w_{\lambda_{0}}>0 & \text { on } \partial B_{r_{0} / 2}(0), \\
w_{\lambda_{0}}=0 \text { and }-\Delta w_{\lambda_{0}} \geq 0 & \text { on } \partial B_{\lambda_{0}}(0) .
\end{array}
$$

By the maximum principle, we have $\Delta w_{\lambda_{0}}<0$ and $w_{\lambda_{0}}>0$ for $r_{0} / 2<|x|<\lambda_{0}$.

Next, by the definition of $\lambda_{0}$, we have two cases to discuss:

Case 1. There exists a sequence $\left\{\lambda_{k}\right\}$ such that $\lambda_{k}<\lambda_{0}$ for all $k$, and further

$$
\lim _{k \rightarrow \infty} \lambda_{k}=\lambda_{0} \quad \text { and } \quad \inf _{\bar{B}_{\lambda_{k}}(0) \backslash\{0\}} w_{\lambda_{k}}<0 .
$$

For $k$ large enough, we have

$$
w_{\lambda_{k}}(x) \geq \frac{1}{2} \quad \text { for } x \in \bar{B}_{r_{0} / 2}(0) \backslash\{0\} .
$$

It follows that there exists $x_{k} \in \bar{B}_{\lambda_{k}}(0) \backslash B_{r_{0} / 2}(0)$ such that

$$
w_{\lambda_{k}}\left(x_{k}\right)=\min _{\bar{B}_{\lambda_{k}}(0) \backslash\{0\}} w_{\lambda_{k}}<0 .
$$

It is clear that $r_{0} / 2<\left|x_{k}\right|<\lambda_{k}$. Hence $\nabla w_{\lambda_{k}}\left(x_{k}\right)$ vanishes and $\Delta w_{\lambda_{k}}\left(x_{k}\right)$ is nonnegative. After passing to a subsequence (still denoted by $x_{k}$ and still converging to $x_{0}$ ), we get

$$
w_{\lambda_{0}}\left(x_{0}\right)=0, \quad \nabla w_{\lambda_{0}}\left(x_{0}\right)=0, \quad \Delta w_{\lambda_{0}}\left(x_{0}\right) \geq 0 .
$$


Thus $\left|x_{0}\right|=\lambda_{0}$. Since $-\Delta w_{\lambda_{0}}(x) \geq 0$ for $x$ near $x_{0}$ if $\left|x_{0}\right|=\lambda_{0}$, Hopf's Lemma yields $(\partial / \partial \eta)\left(w_{\lambda_{0}}\right)\left(x_{0}\right)>0$. This contradicts the equality $\nabla w_{\lambda_{0}}\left(x_{0}\right)=0$, showing that $w_{\lambda_{0}}$ vanishes.

Case 2. Alternatively, suppose there exists a constant $\varepsilon_{0} \in\left(0, \lambda_{0} / 2\right)$ such that, for all $\lambda \geq \lambda_{0}-\varepsilon_{0}$, we have $w_{\lambda} \geq 0$ in $B_{\lambda}(0) \backslash\{0\}$. Then there is a sequence $\left\{\lambda_{k}\right\}$ such that $\lambda_{k}<\lambda_{0}$ for all $k$, and further

$$
\lim _{k \rightarrow \infty} \lambda_{k}=\lambda_{0} \quad \text { and } \quad \inf _{\bar{B}_{\lambda_{k}}(0) \backslash\{0\}}\left(-\Delta w_{\lambda_{k}}\right)<0 .
$$

Now, the relations $w_{\lambda} \geq 0$ on $B_{\lambda}(0) \backslash\{0\}$ and $w_{\lambda}=0$ on its boundary imply that $\partial w_{\lambda} / \partial r \leq 0$ on the boundary. This, together with the formula (4-2) for $-\Delta w_{\lambda}$, shows that $-\Delta w_{\lambda} \geq 0$ on the boundary of the ball.

Then we can apply the maximum principle to show that $-\Delta w_{\lambda} \geq 0$, contradicting the assumptions made.

Remark. By putting Propositions 4.3 and 4.4 together, it is not hard to show that $d=8$, which contradicts the assumption that $d<8$.

Lemma 4.5. For $d \geq 8$, the limit $\alpha=\lim _{|x| \rightarrow \infty}(u(x)+d \log |x|)$ exists and is finite. Furthermore, for $|x| \geq 1$, we have

$$
|u(x)+d \log | x|-\alpha| \leq \frac{C \log |x|}{|x|},\left.\quad|| x\right|^{2} \Delta u+2 d\left|\leq \frac{C}{|x|}, \quad\right| x \cdot \nabla u+d \mid \leq \frac{C}{|x|} .
$$

Proof. Since

$$
u(x)=\alpha-\frac{1}{4 \omega_{4}} \int_{\mathbb{R}^{4}} \log |x-y| e^{u} d y,
$$

we have

$$
|u(x)+d \log | x|-\alpha|=\frac{1}{4 \omega_{4}}\left|\int_{\mathbb{R}^{4}}(\log |x-y|-\log |x|) e^{u} d y\right| \leq \frac{C \log |x|}{|x|},
$$

by elementary calculations. The other two estimates are verified similarly.

Now we consider

$$
w_{\lambda}(x)=u(x)-\left(u\left(\frac{\lambda^{2} x}{|x|^{2}}\right)-8 \log \frac{|x|}{\lambda}\right) .
$$

Clearly, $\Delta^{2} w_{\lambda}=(\exp \psi) w_{\lambda}$.

Proposition 4.6. For $\lambda$ large enough, $w_{\lambda}(x)$ and $-\Delta w_{\lambda}(x)$ are nonnegative for all $x \in B_{\lambda}(0) \backslash\{0\}$. 
Proof. Step 1. There exists $R_{0}$ large enough such that $w_{\lambda} \geq 0$ for all $R_{0} \leq|x| \leq \lambda / 2$. Indeed, by Lemma 4.5,

$$
\begin{aligned}
w_{\lambda} & =u(x)-u\left(\frac{\lambda^{2} x}{|x|^{2}}\right)+8 \log \frac{|x|}{\lambda}=u(x)+8 \log |x|-u\left(\frac{\lambda^{2} x}{|x|^{2}}\right)-8 \log \lambda \\
& =u(x)+d \log |x|+(8-d) \log |x|-u\left(\frac{\lambda^{2} x}{|x|^{2}}\right)-8 \log \lambda \\
& =(u(x)+d \log |x|)-\left(u\left(\frac{\lambda^{2} x}{|x|^{2}}\right)+d \log \frac{\lambda^{2}}{|x|}\right)+(8-2 d) \log \frac{|x|}{\lambda} \\
& \geq(2 d-8) \log 2+(u(x)+d \log |x|)-\left(u\left(\frac{\lambda^{2} x}{|x|^{2}}\right)+d \log \frac{\lambda^{2}}{|x|}\right) \\
& \geq 8 \log 2+O\left(\frac{\log |x|}{|x|}\right),
\end{aligned}
$$

which is positive for $|x|$ large.

Step 2. There exists $R_{1} \geq R_{0}$ such that $w_{\lambda}(x) \geq 0$ and $-\Delta w_{\lambda}(x) \geq 0$ when $R_{1} \leq$ $\lambda / 2 \leq|x| \leq \lambda$, provided that $d>8$. Indeed,

$$
\begin{aligned}
-\Delta w_{\lambda}(x) & =-\Delta u(x)+\Delta\left(u\left(\frac{\lambda^{2} x}{|x|^{2}}\right)\right)-8 \Delta\left(\log \frac{|x|}{\lambda}\right) \\
& =-\Delta u(x)+\frac{\lambda^{4}}{|x|^{4}}(\Delta u)\left(\frac{\lambda^{2} x}{|x|^{2}}\right)-4 \sum_{i=1}^{4}\left(\frac{\lambda^{2} x_{i}}{|x|^{4}} u_{i}\left(\frac{\lambda^{2} x}{|x|^{2}}\right)\right)-\frac{16}{|x|^{2}} \\
& =|x|^{-2}\left(\left(-|x|^{2} \Delta u(x)-2 d\right)+\left(\frac{\lambda^{4}}{|x|^{2}}(\Delta u)\left(\frac{\lambda^{2} x}{|x|^{2}}\right)+2 d\right)\right. \\
& \left.-4\left(\sum_{i=1}^{4}\left(\frac{\lambda^{2} x_{i}}{|x|^{2}} u_{i}\left(\frac{\lambda^{2} x}{|x|^{2}}\right)\right)+d\right)+(4 d-16)\right),
\end{aligned}
$$

which is nonnegative if $R_{1}$ is large enough and $d>8$.

Since $w_{\lambda}(x)=0$ on $|x|=\lambda$ and $w_{\lambda}(x)>0$ on $|x|=\lambda / 2$, from maximum principle, we have $w_{\lambda}(x) \geq 0$ for $R_{1} \leq \lambda / 2 \leq|x| \leq \lambda$ and $R_{1} \geq R_{0}$.

Step 3. There exists $R_{2} \geq R_{1}$ such that for all $\lambda \geq R_{2}, w_{\lambda}(x) \geq 0$ for $x \in B_{R_{0}}(0) \backslash\{0\}$ if $d \geq 8$. Indeed, once again, we have

$$
\begin{aligned}
w_{\lambda}(x) & =u(x)-u\left(\frac{\lambda^{2} x}{|x|^{2}}\right)+8 \log \frac{|x|}{\lambda} \\
& =u(x)-\left(u\left(\frac{\lambda^{2} x}{|x|^{2}}\right)+d \log \frac{\lambda^{2}}{|x|}\right)+(2 d-8) \log \lambda-(d-8) \log |x| .
\end{aligned}
$$


Clearly the condition $|x| \leq R_{0}$ implies $u(x) \geq-C$,

$$
\left|u\left(\frac{\lambda^{2} x}{|x|^{2}}\right)+d \log \frac{\lambda^{2}}{|x|}\right| \leq C \quad \text { for } \lambda \text { large enough, }
$$

and $\log |x| \leq \log R_{0}$. From these relations, together with the condition $2 d-8>0$ and the last formula on the previous page, one obtains $w_{\lambda}(x) \geq 0$ for all $|x| \leq R_{0}$.

This concludes the proof apart from the claim that $-\Delta w_{\lambda} \geq 0$. To show this, we consider the new function $\varphi(x)=|x|^{2}\left(-\Delta w_{\lambda}\right)$. Set $\varepsilon_{r}=\inf _{|x|=r} \varphi(x)$. From Lemma 4.5 and the expression of $-\Delta w_{\lambda}$ in Step 2, we know that $\lim _{r \rightarrow 0} \varepsilon_{r}=0$. Now by a simple calculation, we obtain, for $0<r<\lambda$,

$$
\Delta \varphi(x)-2 \nabla|x|^{2} \cdot \nabla \varphi(x)+|x|^{2}(\exp \psi) w_{\lambda}(x)=0 \quad \text { in } \bar{B}_{\lambda}(0) \backslash B_{r}(0) .
$$

Since $\varphi \geq \varepsilon_{r}$ on $\partial B_{r}(0)$ and $\varphi \geq 0$ on $\partial B_{\lambda}(0)$, from maximum principle, we get

$$
\varphi \geq \min \left\{0, \varepsilon_{r}\right\} \quad \text { on } \bar{B}_{\lambda}(0) \backslash B_{r}(0) .
$$

If we let $r$ go to 0 , we obtain $\varphi \geq 0$ on $\bar{B}_{\lambda}(0) \backslash\{0\}$, which is what we want.

Now we define $u_{b}(x)=u(x+b)$ for any $b \in \mathbb{R}^{4}$ and

$$
w_{\lambda, b}(x)=u_{b}(x)-\left(u_{b}\left(\frac{\lambda^{2} x}{|x|^{2}}\right)-8 \log \frac{|x|}{\lambda}\right) .
$$

We also set

$$
\lambda_{b}=\inf \left\{\lambda>0: w_{\mu, b} \geq 0,-\Delta w_{\mu, b} \geq 0 \text { on } \bar{B}_{\mu}(0) \backslash\{0\} \text { for all } \lambda<\mu<\infty\right\} .
$$

Proposition 4.7. There exists $\bar{b} \in \mathbb{R}^{4}$ such that $\lambda_{\bar{b}}>0$.

Proof. Like that of Proposition 4.3.

Proposition 4.8. Suppose that $\lambda_{b}>0$ for some $b \in \mathbb{R}^{4}$. Then $w_{\lambda, b}(x) 0$ for all $x \in \mathbb{R}^{4}$.

Proof. Without loss of generality, we assume $b=0$. Suppose that $w_{\lambda_{0}}(x) \not \equiv 0$. From the definition of $\lambda_{0}$, we have $w_{\lambda_{0}} \geq 0$ and $-\Delta w_{\lambda_{0}} \geq 0$ in $B_{\lambda_{0}} \backslash\{0\}$, and $\Delta^{2} w_{\lambda_{0}}=e^{\psi} w_{\lambda_{0}}$ in $B_{\lambda_{0}} \backslash\{0\}$. Now

$$
\begin{aligned}
w_{\lambda_{0}}(x) & =u(x)-u\left(\frac{\lambda_{0}^{2} x}{|x|^{2}}\right)+8 \log \frac{|x|}{\lambda_{0}} \\
& =u(x)-\left(u\left(\frac{\lambda_{0}^{2} x}{|x|^{2}}\right)+d \log \frac{\lambda_{0}^{2}}{|x|}\right)+d \log \lambda_{0}^{2}+(8-d) \log |x|-8 \log \lambda_{0},
\end{aligned}
$$

which is greater than or equal to 1 if $|x| \leq r_{0}$ for $r_{0}$ small enough and $d \geq 8$; moreover 


$$
\begin{aligned}
&-\Delta w_{\lambda_{0}}=-\Delta u(x)+\Delta\left(u\left(\frac{\lambda_{0}^{2} x}{|x|^{2}}\right)\right)- 8 \Delta \log |x| \\
&=|x|^{-2}\left(-|x|^{2} \Delta u+\left(\frac{\lambda_{0}^{4}}{|x|^{2}} \Delta u\left(\frac{\lambda_{0}^{2} x}{|x|^{2}}\right)+2 d\right)\right. \\
&\left.-4\left(\frac{\lambda_{0}^{2} x}{|x|^{2}} \cdot(\nabla u)\left(\frac{\lambda_{0}^{2} x}{|x|^{2}}\right)+d\right)+2(d-8)\right),
\end{aligned}
$$

which is nonnegative if $|x| \leq r_{0}$ with $r_{0}$ small enough and $d>8$. Thus, from the maximum principle, we know that $w_{\lambda_{0}} \geq 0$ and $-\Delta w_{\lambda_{0}} \geq 0$ in $B_{\lambda_{0}}(0) \backslash\{0\}$.

Next, by the definition of $\lambda_{0}$, we again have two cases to discuss:

Case 1. There exists a sequence $\left\{\lambda_{k}\right\}$ such that $\lambda_{k} \rightarrow \lambda_{0}, \lambda_{k}<\lambda_{0}$ and

$$
\inf _{\bar{B}_{\lambda_{k}}(0) \backslash\{0\}} w_{\lambda_{k}}<0 .
$$

It is not difficult to see that, for $k$ large enough, we have

$$
w_{\lambda_{k}}(x) \geq \frac{1}{2} \quad \text { for all } x \in \bar{B}_{r_{0} / 2} \backslash\{0\} .
$$

Hence there exists $x_{k} \in \bar{B}_{\lambda_{k}}(0) \backslash B_{r_{0} / 2}(0)$ such that

$$
w_{\lambda_{k}}\left(x_{k}\right)=\min _{\bar{B}_{\lambda_{k}}(0) \backslash B_{r_{0} / 2}(0)} w_{\lambda_{k}}<0 .
$$

Clearly $r_{0} / 2<\left|x_{k}\right|<\lambda_{k}$. Therefore $\nabla w_{\lambda_{k}}\left(x_{k}\right)=0$ and $\Delta w_{\lambda_{k}}\left(x_{k}\right) \geq 0$. After passing to a subsequence (still denoted by $x_{k}$ and still converging to $x_{0}$ ), we get

$$
w_{\lambda_{0}}\left(x_{0}\right)=0, \quad \nabla w_{\lambda_{0}}\left(x_{0}\right)=0, \quad \Delta w_{\lambda_{0}}\left(x_{0}\right) \geq 0 .
$$

Thus $\left|x_{0}\right|=\lambda_{0}$. Since $-\Delta w_{\lambda_{0}}(x) \geq 0$ for $x \in \bar{B}_{\lambda_{0}}(0) \backslash B_{r_{0} / 2}(0)$, by Hopf's Lemma, $\partial w_{\lambda_{0}} / \partial \eta<0$ at $x_{0}$ if $w_{\lambda_{0}} \not \equiv 0$. This contradicts the equality $\nabla w_{\lambda_{0}}\left(x_{0}\right)=0$ since $\partial w_{\lambda_{0}} / \partial \eta=\eta \cdot \nabla w_{\lambda_{0}}\left(x_{0}\right)$.

Case 2. Otherwise, there is a constant $\varepsilon_{0} \in\left(0, \lambda_{0} / 2\right)$ such that $w_{\lambda}(x) \geq 0$ for all $\lambda \geq \lambda_{0}-\varepsilon_{0}$ and all $x \in \bar{B}_{\lambda}(0) \backslash\{0\}$. The same argument used at the end of Proposition 4.4 will bring us the desired contradiction.

Thus by using Propositions 4.7 and 4.8 we can show that $d=8$, which contradicts to assumption that $d>8$. Combining this with the remark after Proposition 4.4, we have $d=8$. In this case, we still work with the Kelvin transformation $v$ of $u$ and can see that Propositions 4.2-4.4 are still valid. The reason is that, for Proposition 4.2, Steps 1 and 2 do not need the assumption $d<8$, Step 3 still holds true with help of Lemma 4.5 and the maximum principle (see the argument given at the end of the proof of Proposition 4.6). Proposition 4.3 holds true in general while Proposition 4.4 will go through without any difficulty. 
Proposition 4.9. For all $b \in \mathbb{R}^{4}$, we have $\lambda_{b}>0$.

Proof. It follows from Proposition 4.3 and Proposition 4.4 that there exists some $\bar{b} \in \mathbb{R}^{4}$ such that $\lambda_{\bar{b}}>0$ and $w_{\lambda_{\bar{b}}^{-1}, \bar{b}}(x)=0$ for all $x \in \mathbb{R}^{4}$. That is, we have

$$
v_{\bar{b}}(x)=v_{\bar{b}}\left(\frac{x}{\lambda_{\bar{b}}^{2}|x|^{2}}\right)-8 \log \left(\lambda_{\bar{b}}|x|\right) .
$$

It follows that

$$
\lim _{|x| \rightarrow \infty}\left(u_{\bar{b}}(x)+8 \log |x|\right)=u_{\bar{b}}(0)-8 \log \lambda_{\bar{b}}=u(\bar{b})-8 \log \lambda_{\bar{b}} .
$$

Suppose that there exists a $b \in \mathbb{R}^{4}$ such that $\lambda_{b}=0$. Then $w_{\lambda, b}(x)=v_{b}(x)-\left(v_{b}\left(\frac{\lambda^{2} x}{|x|^{2}}\right)-8 \log \frac{|x|}{\lambda}\right) \geq 0 \quad$ for all $\lambda>0$ and $x \in B_{\lambda}(0) \backslash\{0\}$.

It follows that

$$
u_{b}\left(\frac{x}{|x|^{2}}\right) \geq u_{b}\left(\frac{x}{\lambda^{2}}\right)-8 \log \frac{\lambda}{|x|} \quad \text { for all } \lambda>0 \text { and } x \in B_{\lambda}(0) \backslash\{0\} .
$$

Fixing $\lambda>0$ and sending $|x|$ to 0 , we have, by (4-4),

$$
u(\bar{b})-8 \log \lambda_{\bar{b}} \geq u_{b}(0)-8 \log \lambda=u(b)-8 \log \lambda .
$$

Now sending $\lambda$ to 0 , we reach a contradiction.

What we have shown so far is this:

Proposition 4.10. Suppose $u$ is a solution of (1-1) satisfying conditions (1-3) and (1-4). Then

$$
\frac{1}{4 \omega_{4}} \int_{\mathbb{R}^{4}} e^{u} d x=8 .
$$

Moreover, for all $b \in \mathbb{R}^{4}$, there exists some $\lambda_{b}>0$ such that

$$
u_{b}(x)=u_{b}\left(\frac{\lambda_{b}^{-2} x}{|x|^{2}}\right)-8 \log \frac{\lambda_{b}}{|x|}
$$

for all $x \in \mathbb{R}^{4}$, where $u_{b}(x)=u(x+b)$.

Proposition 4.11. If $u$ satisfies (4-5), there exist $x_{0}, \lambda>0$ such that

$$
u(x)=4 \log \frac{384^{1 / 4} \lambda}{\left|x-x_{0}\right|^{2}+\lambda^{2}} .
$$


Proof. Set $f(x)=\exp (u(x) / 4)$. From (4-5), we get

$$
\begin{aligned}
f(x+b) & =\exp \left(\frac{1}{4} u(x+b)\right)=\exp \frac{1}{4}\left(u\left(\frac{\lambda_{b}^{-2} x}{|x|^{2}}+b\right)-8 \log \left(\lambda_{b}|x|\right)\right) \\
& =\frac{\lambda_{b}^{-2}}{|x|^{2}} f\left(\frac{\lambda_{b}^{-2} x}{|x|^{2}}+b\right) .
\end{aligned}
$$

A variable change gives

$$
f(x)=\frac{\lambda_{b}^{-2}}{|x-b|^{2}} f\left(\frac{\lambda_{b}^{-2}(x-b)}{|x-b|^{2}}+b\right) .
$$

Now set $A=\lim _{|x| \rightarrow \infty}|x|^{2} f(x)=\lambda_{b}^{-2} f(b)$.

If $A=0$, it is easy to see that $f \equiv 0$, since otherwise $\lambda_{b} \neq 0$, which is impossible.

If $A \neq 0$, we get $f>0$, since $\lambda_{b}^{-2} f(b)=A$. Thus $A>0$. Without loss of generality, we can assume $A=1$. Now for $|x|$ large,

$$
f(x)=\frac{\lambda_{0}^{-2}}{|x|^{2}}\left(f(0)+\frac{\partial f}{\partial x_{i}}(0) \cdot \frac{\lambda_{0}^{-2} x_{i}}{|x|^{2}}+o\left(\frac{1}{|x|}\right)\right)
$$

and

$$
f(x)=\frac{\lambda_{b}^{-2}}{|x-b|^{2}}\left(f(b)+\frac{\partial f}{\partial x_{i}}(b) \frac{\lambda_{b}^{-2}\left(x_{i}-b_{i}\right)}{|x-b|^{2}}+o\left(\frac{1}{|x|}\right)\right) .
$$

Combining these two equations and the assumption $A=1$, we obtain

$$
\frac{\partial f}{\partial x_{i}}(b) f^{-2}(b)=\frac{\partial f}{\partial x_{i}}(0) f^{-2}(0)-2 b_{i} \text {. }
$$

It follows that

$$
\frac{\partial f^{-1}}{\partial x_{i}}(b)=2 b_{i}-\frac{\partial f^{-1}}{\partial x_{i}}(0)
$$

that is,

$$
\frac{\partial}{\partial x_{i}}\left(f^{-1}\right)(b)=\frac{\partial}{\partial x_{i}}\left(|b|^{2}-\frac{\partial f^{-1}}{\partial x_{j}}(0) b_{j}\right) .
$$

Therefore $f^{-1}(b)=\left|b-b_{0}\right|^{2}+\lambda^{2}$ for some $b_{0} \in \mathbb{R}^{4}$ and some constant $\lambda>0$. We conclude that $u(x)=4 \log \left(a /\left(\left|x-x_{0}\right|^{2}+\lambda^{2}\right)\right)$ for some positive number $a$, since $A=1$. Now by a direct check, we see that $a=384^{1 / 4} \lambda$. This completes the proof of Proposition 4.11 and of our main result.

\section{References}

[Caffarelli et al. 1989] L. A. Caffarelli, B. Gidas, and J. Spruck, "Asymptotic symmetry and local behavior of semilinear elliptic equations with critical Sobolev growth", Comm. Pure Appl. Math. 42:3 (1989), 271-297. MR 90c:35075 Zbl 0702.35085 
[Chang and Yang 1995] S.-Y. A. Chang and P. C. Yang, "Extremal metrics of zeta function determinants on 4-manifolds", Ann. of Math. (2) 142:1 (1995), 171-212. MR 96e:58034 Zbl 0842.58011

[Chang and Yang 1997] S.-Y. A. Chang and P. C. Yang, "On uniqueness of solutions of $n$th order differential equations in conformal geometry”, Math. Res. Lett. 4:1 (1997), 91-102. MR 97m:58204 Zbl 0903.53027

[Chen and Li 1991] W. X. Chen and C. Li, "Classification of solutions of some nonlinear elliptic equations”, Duke Math. J. 63:3 (1991), 615-622. MR 93e:35009 Zbl 0768.35025

[Gidas et al. 1979] B. Gidas, W. M. Ni, and L. Nirenberg, "Symmetry and related properties via the maximum principle", Comm. Math. Phys. 68:3 (1979), 209-243. MR 80h:35043 Zbl 0425.35020

[Gilbarg and Trudinger 1983] D. Gilbarg and N. S. Trudinger, Elliptic partial differential equations of second order, 2nd ed., Grundlehren der Mathematischen Wissenschaften 224, Springer, Berlin, 1983. MR 86c:35035 Zbl 0562.35001

[Li and Zhu 1995] Y. Li and M. Zhu, "Uniqueness theorems through the method of moving spheres", Duke Math. J. 80:2 (1995), 383-417. MR 96k:35061 Zbl 0846.35050

[Lin 1998] C.-S. Lin, "A classification of solutions of a conformally invariant fourth order equation in $\mathbf{R}^{n}$ ", Comment. Math. Helv. 73:2 (1998), 206-231. MR 99c:35062 Zbl 0933.35057

Received August 19, 2004. Revised October 14, 2004.

\author{
XINGWANG XU \\ DEPARTMENT OF MATHEMATICS \\ NATIONAL UNIVERSITY OF SINGAPORE \\ 2 SCIENCE DRIVE 2 \\ SINGAPORE 117543 \\ SINGAPORE \\ matxuxw@nus.edu.sg
}

